\title{
A comparison of the effects of reward magnitude and deprivation level on resistance to extinction
}

\author{
T. L. DAVIDSON, ELIZABETH D. CAPALDI, and JANIS L. PETERSON \\ Purdue University, West Lafayette, Indiana 47907
}

\begin{abstract}
In two experiments, effects of varying reward magnitude were compared with the effects of varying deprivation level. Resistance to extinction under low deprivation was found to be an increasing function of deprivation level during acquisition, whether partial or consistent reward was used, and an increasing function of reward magnitude if partial reward was used in acquisition. If consistent reward was used in acquisition, resistance to extinction was inversely related to acquisition reward magnitude. These experiments indicate that all of the effects of reward magnitude and deprivation level on performance cannot be explained by a single incentive mechanism. Two hypotheses that would account for the data are that reward-related stimuli have stronger control over responding than deprivation stimuli and/or that downshifts in reward size produce frustration, but deprivation shifts do not.
\end{abstract}

In several situations, the effects of variations in food deprivation on instrumental performance are highly similar to the effects of variations in reward magnitude (see Mackintosh, 1974, pp. 150-154). Such findings have led to suggestions that deprivation and reward magnitude influence behavior through a common incentive mechanism. Contemporary incentive views (e.g., Bolles, 1975; Mollenauer, 1971) have postulated that deprivation affects incentive motivation by altering the value of relevant rewards. Since reward value is assumed to vary directly with reward magnitude and deprivation level, both of these variables should have similar effects on behavior.

A problem with this approach is that the effects of reward magnitude and deprivation are not always similar. For example, E. D. Capaldi (1972) found increased resistance to extinction with increased acquisition deprivation level, whether consistent or partial reward was used in acquisition, whereas Hulse (1958) and Wagner (1961) reported decreased resistance to extinction with increased magnitude of consistent reward. However, these findings may be more related to between-study differences in experimental conditions than to theoretical considerations. For example, Capaldi (1972) gave extinction 10 or 18 days after acquisition, whereas Hulse and Wagner give extinction $24 \mathrm{~h}$ after acquisition.

The purpose of these experiments was to evaluate the effects of deprivation and reward under the same experimental conditions. In acquisition of a running

This research was supported by Grant MH 23446-01 from the National Institute of Mental Health to Elizabeth D. Capaldi. T. L. Davidson is now at the Department of Psychology, St. Olaf College, Northfield, Minnesota 55057. Reprint requests should be addressed to Elizabeth D. Capaldi, Department of Psychological Sciences, Purdue University, West Lafayette, Indiana 47907. response with rats, two levels of deprivation were combined factorially with two levels of reward magnitude. In extinction, all rats were run under low deprivation. Experiment 1 employed a consistent reward schedule; Experiment 2 used a partial reward schedule. One previous study (Pavlik \& Reynolds, 1963), using consistent reward, employed a similar design. While resistance to extinction was inversely related to reward magnitude, no effect of acquisition deprivation level was found. However, this study varied hours of deprivation in acquisition. There is evidence that the incentive value of food may be more strongly linked to bodyweight manipulations than to variations in hours of deprivation (Renner, Cravens, \& Wooley, 1971). If so, manipulating body weight would be more likely to produce effects similar to those produced by rewardmagnitude manipulations. Hence, body weight was chosen as the method of manipulating deprivation level in the present experiments.

\section{EXPERIMENT 1}

\section{Method}

Subjects. The subjects were 48 naive male albino rats, about 90 days old upon arrival at the laboratory from the Holtzman Company, Madison, Wisconsin.

Apparatus. The apparatus was a runway painted a flat gray throughout, $208.1 \times 10.2 \mathrm{~cm}$, with $22.0-\mathrm{cm}$-high sides covered with $1.3-\mathrm{cm}$ hardware cloth. A $20.3-\mathrm{cm}$-ong floor treadle, suspended over a microswitch, constituted the initial portion of the alley, and when the treadle was depressed by the weight of the rat, a .01-sec clock was started. The clock was stopped (to define start time) and another was started when the rat interrupted a photobeam $5.08 \mathrm{~cm}$ beyond the treadle. The second clock was stopped (run time) and a third one was started when the rat broke the second photobeam, located $132.1 \mathrm{~cm}$ from the first. Interruption of the third photobeam, $38.8 \mathrm{~cm}$ beyond the second, stopped the final clock (goal time). The third photobeam was located $8.5 \mathrm{~cm}$ in front of a teaspoon that was attached to a block of wood and served as a food cup. A manually 
lowered guillotine door, $27.8 \mathrm{~cm}$ from the rear of the goal compartment, served to confine the rat to the goal area. The times from the three clocks were summed to obtain total time.

Procedure. The experiment was run by two experimenters, each of whom ran half the subjects in each group. The design was a 2 by 2 factorial combining body weight ( $80 \%$ vs. $95 \%$ of original ad-lib body weight) during acquisition with reward magnitude ( 2 vs. 22.045 -g Noyes pellets). The groups were designated by the body weight and reward magnitude received in acquisition (e.g., Group $80 \%-2$ was run to 2 pellets under $80 \%$ body weight in acquisition). For their first 11 days in the laboratory, all rats were given ad-lib food and water. The mean weight for each rat on the last 3 of these days was taken as its original ad-lib weight. Rats were randomly assigned to groups on Day 12. Beginning on this day, rats were fed a daily ration designed to bring them to and maintain them at either $80 \%$ (within $79 \%$ $81 \%$ was acceptable) or $95 \%$ (within $94 \%-96 \%$ was acceptable) of their original ad-lib weights. By Day 22, all rats were within their appropriate weight range. On Days 23 and 24, each rat was handled individually for $90 \mathrm{sec}$ and, $15 \mathrm{~min}$ later, was fed the number of pellets it was to receive in acquisition. Acquisition began on Day 25. Each rat received one trial on Day 1 of acquisition, two trials on Day 2, and three trials on Days 3-15. All trials were rewarded, and each rat was removed from the goalbox as soon as all pellets were consumed. After training on Day 15 , rats in Groups $80 \%-2$ and $80 \%-22$ were fed ad lib until they reached $94 \%-96 \%$ of their original ad-lib weights, and then they were given a daily ration to maintain them at that level. Extinction began 9 days after the end of acquisition, lasting for 7 days at 3 trials/day. Throughout the experiment, rats were run in squads of four, composed of one rat from each group, producing an intertrial interval of about $6 \mathrm{~min}$. If a rat failed to traverse an alley section within $60 \mathrm{sec}$, it was gently guided to the next section and $60 \mathrm{sec}$ was recorded for the untraversed portion of the alley. Each squad was fed its daily ration $15 \mathrm{~min}$ after its trials were completed.

\section{Results}

Speeds $(1 / \mathrm{sec})$ in each alley section over all of acquisition, on the last day of acquisition, and over all of extinction were analyzed in separate analyses of variance with experimenter, body weight, and reward magnitude as factors. The results were substantially the same in all alley sections, with differences associated with experimenter being nonsignificant. Thus the results are presented for total speeds only, and the effect of experimenter is not mentioned further.

Over all of acquisition, 22 pellets produced faster running than 2 pellets $[F(1,40)=14.09, p<.001]$ and $80 \%$ body weight produced faster running than $95 \%$ $[F(1,40)=70.74, p<.001]$. The Reward Magnitude by Body Weight interaction was not significant $(F<1)$.

Figure 1 shows the mean total speed for each group on the last day of acquisition and on each day of extinction. As can be seen in Figure 1, running speeds on the last day of acquisition were faster with a 22-pellet reward than with a 2-pellet reward and faster under $80 \%$ than under $95 \%$ body weight. Differences associated with body weight were significant on the last day of acquisition $[\mathrm{F}(1,40)=51.04, \mathrm{p}<.001]$, but differences associated with reward magnitude and the Reward Magnitude by Body Weight interaction were not $[F(1,40)=2.0$ and $F(1,40)<1$, respectively $]$.

Figure 1 also shows that greater resistance to extinction was produced by 2 pellets than by 22 pellets in

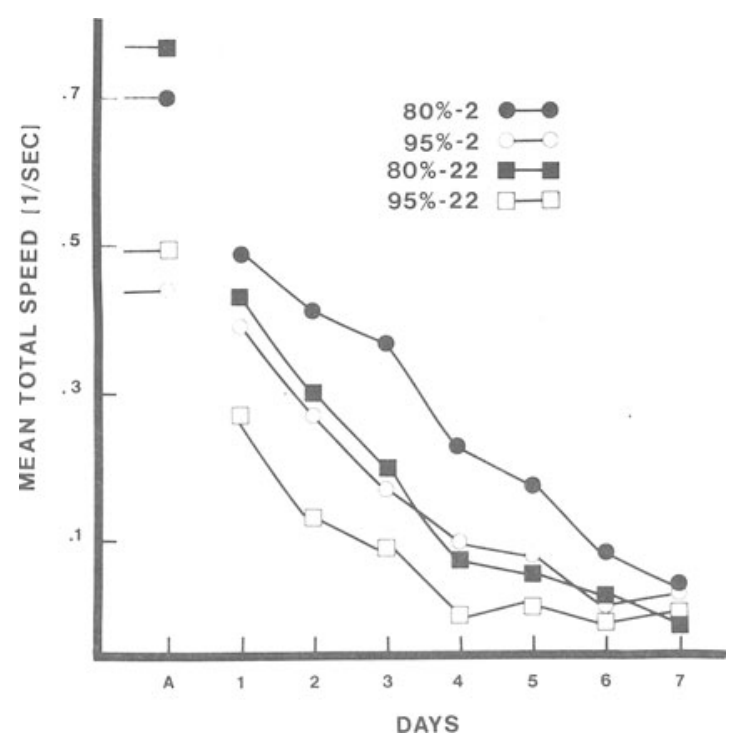

Figure 1. Mean total speed for each of the four groups in Experiment 1 on the last day of acquisition (A) and each day of extinction.

acquisition, and by $80 \%$ body weight compared with 95\%. Extinction differences associated with reward magnitude and body weight were significant $[\mathrm{Fs}(1,40)=$ 25.80 and 28.37 , respectively; ps $<.001]$, but the Reward Magnitude by Body Weight interaction was not significant $[F(1,40)=1.67, p>.10]$. The Reward Magnitude by Days interaction was significant $[F(6,240)$ $=4.87, \mathrm{p}<.001]$, as was the Body Weight by Days interaction $[\mathrm{F}(6,240)=8.19, \mathrm{p}<.001]$, with 22 pellets and $95 \%$ body weight producing a more rapid decline in speed over days than 2 pellets and $80 \%$ body weight.

\section{EXPERIMENT 2}

\section{Method}

Subjects. The subjects were rats of the same description as those in Experiment 1.

Apparatus. The apparatus consisted of a runway painted a flat gray throughout, $144.8 \mathrm{~cm}$ long and $8.9 \mathrm{~cm}$ wide, with $10.2-\mathrm{cm}$-high sides and covered with $1.3-\mathrm{cm}$ hardware cloth. The 27-cm-long startbox was separated from the runway by a metal door that the experimenter dropped by pushing a button. Dropping the door started the first of three .01-sec Standard clocks. Clock 1 was stopped (start time) and Clock 2 started when the rat interrupted a photobeam $30.5 \mathrm{~cm}$ from the door. Clock 2 was stopped (run time) and Clock 3 started when the rat broke a second photobeam, located $43.2 \mathrm{~cm}$ from the first. Interruption of a third photobeam stopped Clock 3 (goal time). The third photobeam was $30.5 \mathrm{~cm}$ beyond the second photobeam and $7.6 \mathrm{~cm}$ in front of a gray block of wood with a $1.9 \mathrm{~cm}$ deep $\times 3.17 \mathrm{~cm}$ from the rear of the goal compartment, served to confine the subject to the goal area. Times from the three clocks were summed to obtain total time.

Procedure. The procedure was identical to that of Experiment 1 , with the following exceptions: Rats were reduced to either $70 \%$ (within $69 \%-71 \%$ ) or $100 \%$ (within $99 \%-101 \%$ ) of their original ad-lib body weights. There was 1 rewarded trial on Day 1 of acquisition, 2 rewarded trials on Day 2, and 4 trials/ day thereafter. On four-trial days, rats received two rewarded 
(R) and two nonrewarded (N) trials. Three schedules of $R$ and $\mathrm{N}$ trials were employed: (A) RNNR, (B) NRRN, (C) NNRR. Half the rats in each group received these schedules in the order $A C B B A C A B C A$, and the remaining half received the order ABCABACBAC. On $\mathbf{R}$ trials, rats were removed from the goalbox as soon as they consumed all of the pellets. On $\mathrm{N}$ trials, rats were removed after a $20-\mathrm{sec}$ confinement. There were 10 days of four trials each in acquisition. In extinction, all rats were given 4 trials/day for 7 days under $100 \%$ ad-lib weight. Fourteen days were required to adjust weights prior to handling and pellet experience, and it took 15 days to adjust weights to $100 \%$ ad lib after acquisition. During the experiment, one rat in Group 100\%-22 died, and its data were discarded.

\section{Results}

The form of the analysis of variance was the same as in Experiment 1, and as in Experiment 1, results were substantially the same in each alley section and for each experimenter. Although rats were run for 10 four-trial days in acquisition, no times were obtained on Days 7 and 10 due to an equipment failure.

In acquisition, there were significant differences associated with body weight $[F(1,31)=44.76, p<.001]$ and reward magnitude $[\mathrm{F}(1,31)=4.33, \mathrm{p}<.05]$, with rats running faster under $70 \%$ than under $100 \%$ body weight and faster with 22- than with 2-pellet reward.

Figure 2 shows the mean total speeds for each group on the next-to-last day of acquisition (the last day for which speeds were available) and on each day of extinction. As can be seen in Figure 2, running speeds on the next-to-last day of acquisition were faster the higher the deprivation level and the larger the reward magnitude. Analysis of variance found the difference associated with body weight to be significant $[F(1,31)=6.31$,

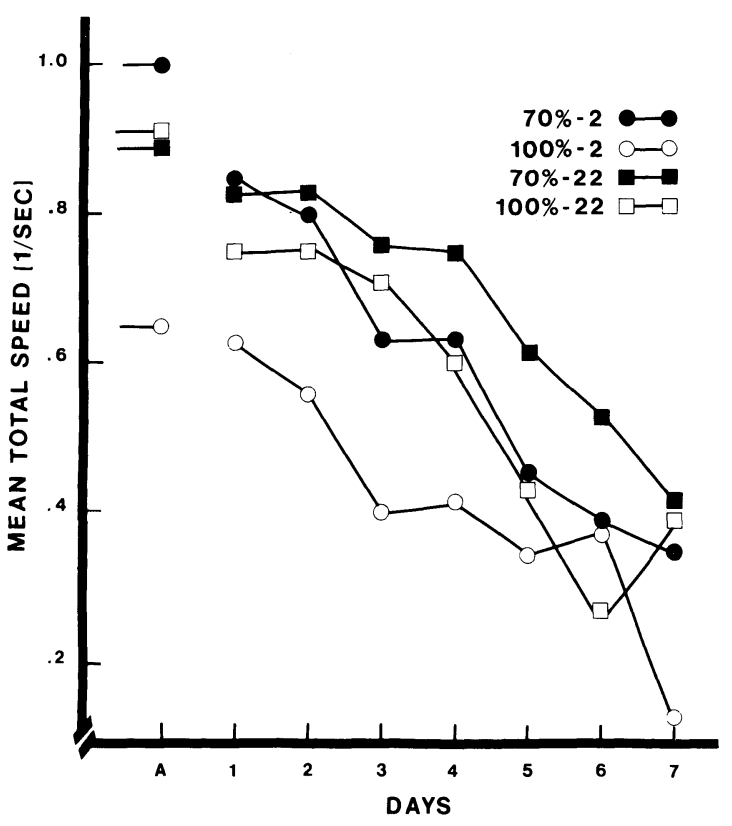

Figure 2. Mean total speed for each of the four groups in Experiment 2 on the next-to-last day of acquisition (A) and each day of extinction. $\mathrm{p}<.02]$, but the difference associated with reward magnitude was not $[F(1,31)=1.36, p>.20]$.

As is also shown in Figure 2, resistance to extinction was greater the larger the reward magnitude and the higher the deprivation level in acquisition. Differences associated with reward magnitude were significant in the start and run [smallest $F(1,31)=4.34, p<.05$, in run] and just missed significance in total $[\mathrm{F}(1,31)=$ $4.00, p=.051]$. Differences associated with body weight were significant in start, goal, and total [smallest $F(1,31)=4.23, p<.05$, in goal] . The Body Weight by Reward Magnitude interaction was not significant in any alley section or in total $\left(F_{s}<1\right)$.

\section{DISCUSSION}

The results of Experiments 1 and 2 show that when evaluated under the same experimental conditions, resistance to extinction following partial reward training is an increasing function of both acquisition reward and deprivation level. However, while resistance to extinction is an increasing function of acquisition deprivation level when consistent reward is used, resistance to extinction decreased with increased magnitude of consistent reward.

These results suggest that the effects of deprivation level and reward magnitude on instrumental performance are not mediated completely by a single common incentive mechanism. This conclusion is also suggested by other data we have presented (e.g., E. D. Capaldi \& Friedman, 1976; E. D. Capaldi, Smith, \& White, 1977).

The relationship between magnitude of consistent reward and resistance to extinction has been attributed to betweengroups differences in stimulus generalization decrement produced by changes in internal reward-related stimuli (e.g., E. J. Capaldi, 1967) and/or to differences between groups in the frustration produced by extinction (e.g., Amsel, 1958). If the influence of stimulus generalization decrement or frustration is reduced in the case of deprivation shifts relative to reward shifts, the difference between the effect of deprivation and the effect of reward magnitude obtained here could be accounted for. For example, if deprivation stimuli exert weaker control over instrumental responding than internal reward-related cues do, changing deprivation level will produce less stimulus generalization decrement than will changing reward magnitude: Thus, resistance to extinction will be greater for groups shifted from high to low deprivation than for groups shifted from large reward to nonreward. A similar associative analysis was offered by Likely, Little, and Mackintosh (1971) with respect to their finding that resistance to extinction was inversely related to magnitude of food reward, but directly related to magnitude (i.e., concentration) of sucrose reward. Likely et al. suggested that changing sucrose concentration and its stimulus aftereffects produced less stimulus generalization decrement and, hence, less disruption of performance than did changing magnitude of food reward and its stimulus consequences. From the viewpoint of a frustration theorist, it is possible that deprivation downshifts, unlike reward magnitude reductions, do not produce frustration. Thus, frustration would not reduce resistance to extinction in groups shifted from high to low deprivation as it would in groups shifted from large reward to nonreward.

The finding that deprivation and reward magnitude have similar effects on extinction performance following partial reward training is compatible with both of the above interpretations. To the extent that partial reward, relative to consistent reward, training reduces stimulus generalization decrement or frustration, the effects of decreases in deprivation level and reductions in reward magnitude should be similar. Further, the finding that resistance to extinction increased with acquisition 
deprivation level can be attributed to greater habit strength produced by reward received under high deprivation than by reward received under low deprivation (e.g., E. D. Capaldi \& Hovancik, 1973). This interpretation is compatible with both the associative and the frustration point of view.

\section{REFERENCES}

Amsel, A. The role of frustrative nonreward in noncontinuous situations. Psychological Bulletin, 1958, 55, 102-119.

Bolles, R. C. Theory of motivation (2nd ed.). New York: Harper \& Row, 1975.

Capaldi, E. D. Resistance to extinction in rats as a function of deprivation level and schedule of reward in acquisition. Journal of Comparative and Physiological Psychology, 1972, 79, 90-98.

Capaldi, E. D., \& Friedman, F. Deprivation and reward stimuli as compound stimuli. Learning \& Motivation, 1976, 7, 17-30.

Capaldi, E. D., \& Hovancik, J. R. Effects of previous body weight level on rats' straight-alley performance. Journal of Experimental Psychology, 1973, 97, 93-97.

Capaldi, E. D., Smith, N. S., \& White, L. Control of reward expectancies by drive stimuli. Journal of Experimental Psychology: Animal Behavior Processes, 1977, 3, 229-235.

Capaldi, E. J. A sequential hypothesis of instrumental learning. In K. W. Spence \& J. T. Spence (Ed.), The psychology of learning and motivation (Vol. 1). New York: Academic Press, 1967.
Hulse, S. H. Amount and percentage of reinforcement and duration of confinement in conditioning and extinction. Journal of Experimental Psychology, 1958, 56, 48-57.

Likely, D., LitTle, L., \& Mackintosh, N. J. Extinction as a function of magnitude and percentage of food or sucrose reward. Canadian Journal of Psychology, 1971, 25, 130-137.

Mackintosh, N. J. Psychology of animal learning. London: Academic Press, 1974.

Mollenauer, S. O. Shift in deprivation level: Different effects depending on amount of preshift training. Learning and Motivation, 1971, 2, 58-66.

Pavlik, W. B., \& ReYnolds, W. F. Effects of deprivation schedule and reward magnitude on acquisition and extinction performance. Journal of Comparative and Physiological Psychology, 1963, 56, 452-455.

Renner, K. E., Cravens, R. W., \& Wooley, O. W. Relative utility of food reward as a function of cyclic deprivation or body weight loss in albino rats. Journal of Experimental Psychology, 1971, 90, 102-112.

WAGNER, A. R. Effects of amount and percentage of reinforcement and number of acquisition trials on conditioning and extinction. Journal of Experimental Psychology, 1961, 62, 234242.

(Received for publication November 5, 1981.) 